\title{
La saliva como alternativa de prueba diagnóstica en la detección de COVID-19
}

\author{
Saliva as an alternative diagnostic test in the detection of COVID-19 \\ Pamela J. Castañeda-Hurtado ${ }^{a}$, Horacio Islas-Granillo ${ }^{b,}$ José de Jesús Navarrete-Hernández, ${ }^{c}$
}

\begin{abstract}
:
The World Health Organization (WHO) announced that the 2019 coronavirus disease (COVID-19) epidemic, originating in Wuhan, China, has become a major public health challenge of international concern not only to China but also for countries around the world, within the clinical manifestations of this disease, fever, dry cough and dyspnea have been reported as the most prevalent, followed by myalgia, sputum, sore throat, headache, diarrhea and general malaise; in connection with the current emergency situation, it is very important to prepare rapid and accurate diagnostic test methods for SARS-CoV-2 for the identification of suspected patients with the aim of controlling the outbreak in the community and in hospitals; recent studies have shown that saliva could be a valuable sample for diagnosis considered as a non-invasive procedure that has adequate precision and reliability regarding the detection of SARSCoV-2 according to the RT-PCR technique, which makes it a valid alternative for the diagnosis and monitoring of the SARS-CoV-2 viral load, providing a cost-effective and convenient platform, with a relatively minimal risk of cross infection, thus considering it as an ideal diagnostic biofluid.
\end{abstract}

Keywords:

Coronavirus, public health, diagnostic test, saliva

Resumen:

La Organización Mundial de la Salud (OMS) anunció que la epidemia de la enfermedad por coronavirus 2019 (COVID-19), originada en Wuhan, China, se ha convertido en un importante desafío de salud pública de interés internacional no solo para China sino también para países de todo el mundo, dentro de las manifestaciones clínicas de esta enfermedad, se ha reportado fiebre, tos seca y disnea como las más prevalentes, seguido de mialgia, esputo, dolor de garganta, dolor de cabeza, diarrea y malestar general; en relación con la situación de emergencia actual, es muy importante preparar métodos de prueba de diagnóstico rápidos y precisos del SARS-CoV2 para la identificación de pacientes sospechosos, con el objetivo de controlar el brote en la comunidad y en los hospitales; estudios recientes han demostrado que la saliva podría ser una muestra valiosa para el diagnóstico considerado como un procedimiento no invasivo que tiene la precisión y confiabilidad adecuada con respecto a la detección del SARS-CoV-2 según la técnica de RT-PCR., lo que la convierte en una alternativa válida para el diagnóstico y el monitoreo de la carga viral de SARS-CoV-2, proporcionando una plataforma rentable y conveniente, con un riesgo relativamente mínimo de infección cruzada, considerándola así como un biofluido diagnóstico ideal.

Palabras Clave:

Coronavirus, salud pública, prueba diagnóstica, saliva

\section{Introducción}

La Organización Mundial de la Salud (OMS) anunció que la epidemia de la enfermedad por coronavirus 2019 (COVID-19), originada en Wuhan, China, se ha convertido

\footnotetext{
"a Autor de Correspondencia, Pamela Josuary Castañeda Hurtado, Universidad Autónoma del Estado de Hidalgo, México, https://orcid.org/0000-0003-2324-4078, Email: ca429497@uaeh.edu.mx

b Horacio Islas Granillo, Universidad Autónoma del Estado de Hidalgo, México, https://orcid.org/0000-0002-9706-1218, Email: hislasg@uaeh.edu.mx

c José de Jesús Navarrete Hernández, Universidad Autónoma del Estado de Hidalgo, México, https://orcid.org/0000-0002-7923-8737, Email: josedejesus_navarrete@uaeh.edu.mx
} 
en un importante desafío de salud pública de interés internacional ${ }^{1,2}$ no solo para China sino también para países de todo el mundo. El agente causal se identificó a partir de muestras de hisopos de garganta realizadas por el Centro Chino para el Control y la Prevención de Enfermedades el 7 de enero de 2020 siendo denominado Coronavirus 2 del Síndrome Respiratorio Agudo Severo (SARS-CoV-2). Posteriormente, la enfermedad fue nombrada COVID-19 por la OMS. ${ }^{3}$

La literatura científica biomédica de las últimas semanas se ha centrado sustancial e intensamente a responder las interrogantes que surgen respecto al SARSCoV-2 y la enfermedad de este coronavirus, que augura un largo tiempo en que el mundo deberá no solo convivir con el virus, sino también, buscar las alternativas para su curación o, en primeras instancias, su detección efectiva entre los casos sospechosos ya que prácticamente toda la población mundial está expuesta de forma natural al virus, el solo hecho de convivir en sociedad y el contacto regular de los grupos humanos en sí mismo, hace que el riesgo de contagio sea latente. ${ }^{4}$

El virus SARS-CoV-2 puede sobrevivir en una variedad de superficies, incluso en plástico durante $72 \mathrm{~h}$, en acero inoxidable durante $48 \mathrm{~h}$, en cobre durante $8 \mathrm{~h}$, cartón después de $24 \mathrm{~h},{ }^{5}$ y en la capa exterior de una máscara quirúrgica podría estar presente un nivel detectable de virus infeccioso el día $7,{ }^{6}$ sujeto a una humedad y temperatura favorables.

Dentro de las manifestaciones clínicas de esta enfermedad, se ha reportado fiebre, tos seca y disnea como las más prevalentes, seguido de mialgia, esputo, dolor de garganta, dolor de cabeza, diarrea y malestar general, ${ }^{2,4}$ algunos también pueden presentar dificultad para respirar, fatiga y otros síntomas atípicos, como dolor muscular, confusión, y vómito, ${ }^{7,8}$ también se han detectado otros síntomas entre los casos confirmados, como anosmia (pérdida del olfato) y ageusia (pérdida del gusto), ${ }^{9}$ en casos graves pueden provocar neumonía, insuficiencia renal e incluso la muerte. ${ }^{10}$

Entre los pacientes hospitalizados en Wuhan, alrededor de un cuarto a un tercio desarrollaron complicaciones graves, como síndrome de dificultad respiratoria aguda, arritmia y shock, por lo que fueron trasladados a la unidad de cuidados intensivos . ${ }^{11-13}$

Entre los pacientes que se sometieron a tomografía computarizada (TC) de tórax, la mayoría mostró neumonía bilateral, siendo la opacidad en vidrio deslustrado y las sombras irregulares bilaterales los patrones más comunes. ${ }^{8,13}$ En general, la edad avanzada y la existencia de comorbilidades subyacentes (p. Ej., Diabetes, hipertensión y enfermedad cardiovascular) se asociaron con un peor pronóstico. ${ }^{13,14}$

Pero se han informado casos asintomáticos, casos sin presencia de fiebre e incluso se han reportado transmisiones antes de la aparición de signos y síntomas, de hecho, estudios han demostrado que el 41,6\% de los casos con COVID- 19 son asintomáticos ${ }^{15}$ y pasan completamente desapercibidos, sin embargo, pueden servir como reservorio y contribuir a la propagación de la enfermedad. ${ }^{16}$

Según los hallazgos de la investigación genética y epidemiológica, parece que el brote de COVID-19 comenzó con una sola transmisión de animal a humano, seguida de una propagación sostenida de humano a humano. ${ }^{17,18}$ Los estudios han demostrado que los Coronavirus pueden transmitirse de persona a persona a través del contacto directo o indirecto, mediante gotas gruesas o pequeñas de secreciones provenientes del tracto respiratorio. ${ }^{7}$ Karl Georg Friedrich Wilhelm Flügge, en la década de 1890, demostró que las gotitas que se expulsan de forma inadvertida por la boca y nariz al realizar acciones como toser o estornudar, incluso al hablar en voz baja, o simplemente al espirar, pueden transportar diversos microbios patógenos, tales como bacterias y virus. La mayoría de las microgotas provienen de la saliva, y pueden caer sobre objetos o superficies que rodean a la persona, y de esta forma, los demás pueden contaminarse si tocan estos objetos o superficies y, a su vez, con las manos contaminadas al tocarse los ojos, la nariz o la boca podrán darle entrada al virus a todo el sistema orgánico. ${ }^{19}$

El período de incubación en pacientes susceptibles a COVID-19 es de 1 a 14 días, con un promedio de 3 a 7 días. ${ }^{11}$ A partir de los datos existentes, el virus SARS-CoV-2 se puede detectar en múltiples fuentes, incluido el tejido gastrointestinal, ${ }^{20}$ las lágrimas, ${ }^{21}$ las heces, ${ }^{22}$ la sangre, ${ }^{23}$ y la saliva ${ }^{16,24-26}$ de los pacientes con COVID-19.

El acto de hablar, toser, estornudar, que son acciones respiratorias propias de nuestro organismo y que generan liberación de aerosoles, ha tomado la connotación negativa socialmente dentro del contexto mundial actual, ya que como es bien sabido es en estas circunstancias en las que el virus puede potencialmente ser inoculado entre personas, y que es prácticamente la vía en que particularmente todos los virus de implicancia a nivel respiratorio pueden llegar de un huésped a otro en determinadas circunstancias.

Estas mismas características infectivas ha llevado a movilizar a los sistemas de salud públicos a promocionar y educar a la población en medidas de higiene rigurosas, uso generalizado de equipos de protección de barrera personal, distanciamiento y aislamiento sistemático tanto individual como comunitario y movilización de personal logístico idóneo para la sanitización, orden y organización efectiva de ciudades, edificios e instalaciones. ${ }^{27}$ 


\section{Actuales pruebas diagnósticas de COVID-19}

Según los datos de secuenciación del genoma actualmente disponibles, el SARS-CoV-2 es un nuevo virus de ARN monocatenario de sentido positivo envuelto zoonótico de la familia Coronaviridae que se identificó en la década de $1960 .{ }^{28}$ EI SARS-CoV-2 comparte el 96\% de identidad con un coronavirus de murciélago (BatCoVRaTG13), ${ }^{29}$ el $91,02 \%$ con pangolín-CoV ${ }^{30}$ y el $79,5 \%$ con el coronavirus del síndrome respiratorio agudo severo (SARS-CoV), ${ }^{31}$ respectivamente, a nivel de genoma completo. El coronavirus original del síndrome respiratorio agudo severo (SARS-CoV), ${ }^{32}$ el síndrome respiratorio coronavirus de Oriente Medio (MERS-CoV), ${ }^{33}$ y el SARSCoV-2 pertenecen a un género de coronavirus $\beta$ que infecta a mamíferos y humanos.

Actualmente, el enfoque de COVID-19 es controlar la fuente de infección; utilizar medidas de prevención y control de infecciones para reducir el riesgo de transmisión; y proporcionar diagnóstico temprano, aislamiento y atención de apoyo a los pacientes afectados. ${ }^{13}$ En relación con la situación de emergencia actual, es muy importante preparar métodos de prueba de diagnóstico rápidos y precisos del SARS-CoV-2 para la identificación de pacientes sospechosos con el objetivo de controlar el brote en la comunidad y en los hospitales. ${ }^{34}$ Aparte del nivel de sensibilidad y especificidad de las técnicas de laboratorio, es muy importante seleccionar los sitios apropiados para recolectar muestras. ${ }^{35}$

El diagnóstico 2019-nCoV se realiza con una combinación tanto de exámenes clínicos, radiológicos y moleculares, donde la prueba de reacción en cadena de la polimerasa con transcriptasa inversa (RT-PCR) ha sido el examen de elección para el análisis de material genético viral de muestras extraídas del tracto respiratorio superior. ${ }^{2}$ Los hisopos nasofaríngeos y orofaríngeos son los tipos de muestra recomendados, la técnica consiste en incluir un hisopo a través de las narinas del paciente hasta encontrar el techo del órgano y hurgar sobre el tejido de modo de embeber el instrumento de mucosidad de la zona. $^{36,37}$

A pesar de que RTPCR es una prueba diagnóstica efectiva, no siempre logra un diagnóstico certero, dado que múltiples factores pueden afectar el resultado de esta prueba como: un muestreo clínico incorrecto, la fuente de muestras (tracto respiratorio superior o inferior), el tiempo de la muestra (periodo diferente del desarrollo de la enfermedad), el rendimiento de los kits de detección, baja carga viral del paciente. ${ }^{38}$
Es por esto que un solo resultado negativo de la prueba de RT-PCR de pacientes sospechosos no excluye la infección por COVID-19, de hecho, se ha informado en estudios que la tasa positiva total de RT-PCR para muestras de hisopos de garganta era de aproximadamente $30 \%$ a $60 \%$; la baja sensibilidad de RTPCR implica que muchos pacientes con COVID-19 pueden no ser identificados, pudiendo seguir siendo fuentes de contagio $v$ y no recibir el tratamiento adecuado a tiempo. ${ }^{38}$

Sin embargo, la recolección de estos tipos de muestras requiere un contacto cercano entre los trabajadores de la salud y los pacientes, lo que plantea un riesgo de transmisión. Además, la recolección de muestras nasofaríngeas u orofaríngeas causa molestias y puede causar sangrado especialmente en pacientes con trombocitopenia, por lo tanto, los hisopos nasofaríngeos no son deseables para el monitoreo en serie de la carga viral. 24,35

\section{La saliva como alternativa de prueba diagnóstica}

En primer lugar, el virus comúnmente se encuentra en el tracto respiratorio inferior y superior ingresando y relacionándose con la cavidad oral a través del traspaso de secreciones de manera bidireccional. En segundo lugar, el virus circulante en el torrente sanguíneo puede acceder a la boca a través del líquido gingival crevicular, un exudado específico de la cavidad oral que contiene proteínas locales derivadas de la matriz extracelular y proteínas derivadas del suero (10). Finalmente, la tercera forma en que el COVID-19 puede presentarse en la cavidad oral es mediante la colonización e infección de las glándulas salivales mayores y menores con la posterior liberación de partículas en la saliva a través de los conductos salivales. ${ }^{2}$

La saliva humana es un fluido corporal distintivo que es producido por las glándulas salivales. La saliva se compone principalmente de agua (94-99\%) con moléculas orgánicas que representan casi el $0,5 \%$ y las inorgánicas el 0,2\%. Desempeña un papel importante en la digestión de los alimentos, lubricando la mucosa bucal, limpiando y preservando la cavidad bucal e influyendo en la homeostasis de la cavidad bucal. El flujo diario total de saliva en un adulto normal mide, en promedio, entre 500 ml y 1,5 L. ${ }^{39}$ Además de las excretas de las glándulas salivales, la saliva también incluye partículas de alimentos, elementos del suero, microorganismos orales y sus metabolitos, glóbulos blancos y células epiteliales exfoliadas.

Hasta ahora, se han detectado más de 700 especies microbianas en la saliva, muchas de las cuales están relacionadas con enfermedades orales y 
sistémicas. La saliva no solo ofrece un nicho ecológico para la colonización y el desarrollo de microorganismos orales, sino que también previene el crecimiento excesivo de patógenos particulares para preservar la homeostasis de la cavidad bucal. ${ }^{40}$

El hecho de que se necesite de forma urgente la detección de los pacientes infectados con el mínimo riesgo para el personal de salud, al exponerse al caso sospechoso, hace necesaria la inclusión de nuevas alternativas de toma de muestras. ${ }^{16}$ A causa de la pandemia de COVID-19 en curso se ha generado una escasez de hisopos nasofaríngeos (NPS), medios de transporte viral, y kits para la extracción de ARN viral lo que compromete la cantidad de pruebas que se pueden realizar en muchas partes del mundo, ${ }^{41}$ se requiere la búsqueda de muestras de diagnóstico alternativas, como la saliva, que cobra un rol preponderante, al ser considerada como método de detección positiva de otros virus pudiendo representar un instrumento válido en el diagnóstico de COVID-19, proporcionando información sobre la evolución clínica de la enfermedad. ${ }^{16,27,42}$

La saliva es un fluido complejo que contiene varias enzimas, electrolitos, proteínas, ácidos nucleicos, componentes antimicrobianos, hormonas, citocinas y anticuerpos.

En los animales, el ARN del coronavirus, llamado virus de la diarrea epidémica porcina (PEDV), puede detectarse en la saliva de los cerdos infectados mediante PCR en tiempo real al mismo tiempo pero en concentraciones más altas que en el suero. ${ }^{43,44}$ En otras especies de virus como el flavivirus Zika, el virus se puede detectar en la saliva de pacientes humanos infectados con mayor sensibilidad que en el suero. ${ }^{45}$ Además, la saliva se utiliza ampliamente en el diagnóstico de la infección por citomegalovirus. ${ }^{46}$

Su composición refleja virtualmente todo el estado de salud y enfermedad en un cuerpo y tiene el potencial de ser un medio de diagnóstico para una amplia gama de enfermedades, como en la detección de enfermedades periodontales, evaluaciones de riesgo de caries, mama cáncer, cánceres orales, enfermedades de las glándulas salivales, VIH y mucho más. ${ }^{47}$

Ha sido utilizada para la detección de anticuerpos frente al virus de la rubéola, parotiditis y sarampión. En los recién nacidos la presencia de $\lg A$ es un excelente marcador frente a la infección por rotavirus. Algunas investigaciones sugieren que la reactivación de las infecciones por virus del herpes tipo-1 está relacionada con la patogenia de la parálisis de Bell y que la detección mediante PCR del virus en saliva sería un método adecuado para la detección precoz de las reactivaciones de esta enfermedad. ${ }^{48}$
Es un espécimen prometedor no invasivo para el diagnóstico, monitoreo y control de infecciones en pacientes con infección 2019-nCoV. Las muestras de saliva se pueden proporcionar fácilmente, no se requieren procedimientos invasivos y su recolección puede minimizar en gran medida la posibilidad de exponer a los trabajadores de la salud a 2019-nCoV.

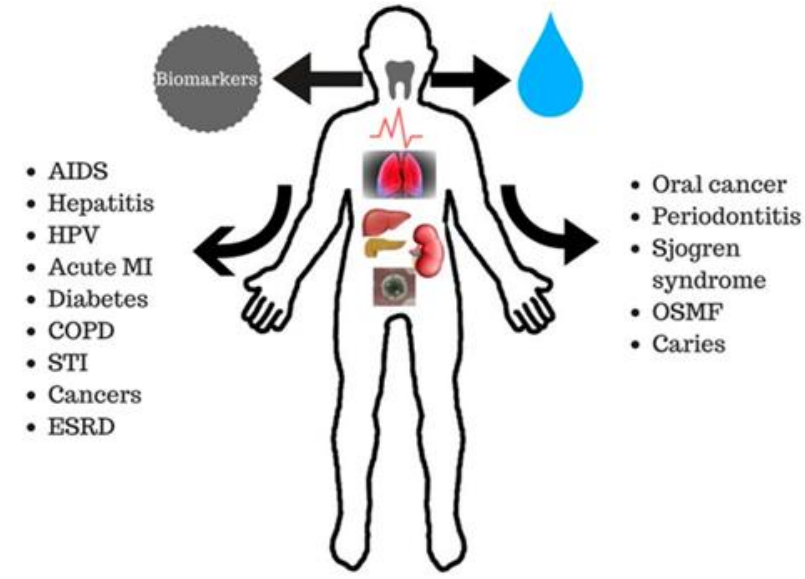

Figura 1. Representación de la detección de diversas enfermedades orales $y$ sistémicas mediante biomarcadores salivales. Síndrome de inmunodeficiencia adquirida (SIDA), Virus del papiloma humano (VPH), Infarto de miocardio (IM), Enfermedad pulmonar obstructiva crónica (EPOC), Infección de transmisión sexual (ITS), Insuficiencia renal en etapa terminal (ESRD) y Fibrosis mucosa escamosa oral (OSMF). ${ }^{47}$

Es fácilmente aceptado por los pacientes ya que no es doloroso ni estresante. Por tanto, puede utilizarse para muestreos seriados y en estudios epidemiológicos 0 de gran escala, siendo especialmente ventajoso en determinadas poblaciones, como los niños. ${ }^{49}$

Se ha demostrado previamente que la saliva tiene una alta tasa de concordancia de más del $90 \%$ con muestras nasofaríngeas en la detección de virus respiratorios, incluidos los coronavirus, esto de acuerdo con las pruebas para 2019-nCoV realizadas en Hong Kong, en la Subdivisión de Servicios de Laboratorio de Salud Pública para los pacientes que cumplían los criterios de notificación o los criterios de vigilancia mejorada, ${ }^{50}$ el SARS-CoV se pudo detectar en la saliva con títulos elevados.

To et al., en 2017 y Wang et al. en 2004 ${ }^{51,52}$ demostraron una alta carga viral con presencia de ARN del virus SARS-CoV en muestras orofaríngeas y de saliva, reafirmando la hipótesis de usar la saliva como un posible reservorio diagnóstico para la epidemia actual de COVID19.

Otro estudio pionero realizado en el Hospital Central de Xiangtan analizó los tiempos de conversión de ácido nucleico de SARS-CoV-2 de diferentes muestras 
obtenidas de pacientes con COVID 19, las cuales fueron sometidas a RT-PCR, la tasa de conversión positiva de ácido nucleico para la saliva fue del $78,1 \%$, siendo muy superior a otras muestras como las lágrimas y la orina. ${ }^{53}$

Del mismo modo se realizó una evaluación de muestras de saliva orofaríngeas en donde el ARN del SARS-CoV-2 fue detectado en el $87 \%$ de pacientes, además de demostrar que la carga viral fue más alta durante la primera semana del inicio de los síntomas. ${ }^{24} \mathrm{En}$ febrero de 2020 Kelvin Kai-Wang et al., lograron detectar 2019-nCoV en las muestras de saliva en 11 de 12 pacientes estudiados. El cultivo viral demostró la presencia de virus vivos en la saliva de 3 pacientes. ${ }^{25}$

En febrero de 2020 Shufa Zheng mostró que la tasa de detección de SARS-CoV-2 basada en muestras de esputo $(95,65 \%)$ y saliva $(88,09 \%)$ fue significativamente mayor que la de los hisopos nasales o de garganta. ${ }^{54}$

El estudio de Chen L, Zhao J, Peng J, Li X, Deng $X$, Geng Z, et al..$^{55}$ demostró que entre los 13 pacientes cuyas pruebas de frotis orofaríngeas fueron positivas, 4 casos también fueron positivos para sus muestras de saliva.

Un estudio realizado en pacientes chinos, informó los resultados sobre la base de un total de 1846 muestras respiratorias (1178 muestras de saliva y 668 muestras de esputo) de 96 casos confirmados ${ }^{56}$ informando que el SARS-CoV-2 se detectó en los 96 pacientes analizando muestras respiratorias.

Estudios realizados en Australia e Italia entre pacientes confirmados con COVID-19, informaron una tasa de detección de 84,6 y $100 \%$, respectivamente, sobre la base de muestras de saliva. ${ }^{16,57}$

En abril de 2020 Jin Gu Yoon et al..$^{58}$ demostraron que la carga viral del SARS-CoV-2 es constantemente alta en la saliva; siendo relativamente más alto que en la orofaringe durante la etapa inicial de COVID-19.

Uno de los estudios incluidos en esta revisión es un informe de caso relativo a un neonato confirmado con SARS-CoV-2, en el cual fue detectado el virus en todas las muestras clínicas del neonato, incluida la sangre, la orina, las heces y la saliva junto con las muestras del tracto respiratorio superior. ${ }^{59}$

Hace algunas semanas los investigadores de la Universidad Rutgers recibieron luz verde de parte del Gobierno de EE.UU. para los primeros test de saliva que ayuden a diagnosticar COVID-19 (IgM-lgG); ${ }^{19}$ en abril de 2020, fue aprobada por la Food and Drugs Administration de los Estados Unidos, el comienzo de las pruebas clínicas para la detección de la infección por el virus Síndrome respiratorio agudo severo coronavirus 2 (SARS-CoV-2) y síndrome coronavirus 2 (SARS-CoV-2) a través de muestras de saliva en los centros de salud que tienen convenio con la Universidad de Rutgers, institución que diseño el test. ${ }^{27}$

Un estudio realizado en la UNAM comparó los resultados de RT-qPCR de 253 muestras pareadas obtenidas de saliva y frotis de pacientes ambulatorios; encontrando una muy buena correlación de resultados entre los dos tipos de muestras, proponiendo que el muestreo de saliva representa una excelente alternativa que facilitará el muestreo y diagnóstico de un mayor número de personas a un costo reducido. ${ }^{41}$

Estudios recientes han demostrado que la saliva podría ser una muestra valiosa para el diagnóstico de infecciones por virus respiratorios en comparación con los hisopos nasofaríngeos, ${ }^{58}$ considerada como una muestra no invasiva tiene la precisión y confiabilidad adecuadas con respecto a la detección del SARS-CoV-2 según la técnica de RT-PCR(35).

\section{Ventajas del test salival}

Existen ventajas en el uso de muestras de saliva como prueba diagnóstica en comparación con los otros tipos de prueba actualmente utilizados: en primer lugar constituye un tipo de procedimiento no invasivo, a diferencia de los hisopos nasofaríngeos y orofaríngeos, que, a pesar de ser efectivos, son métodos dolorosos e incómodos para el paciente, los cuales pueden generar complicaciones durante la toma de la muestra como hematomas, erosiones de la mucosa y sangrado; situación problemática para aquellos pacientes con enfermedades hemorrágicas tales como trombocitopenia, ${ }^{10.34}$ otra ventaja es el método de recolección de saliva ya que este podría efectuarse incluso por el mismo paciente fuera de los hospitales donde no se dispone de salas de aislamiento de infecciones transmitidas por el aire, como en clínicas para pacientes ambulatorios 0 en la comunidad. ${ }^{25}$

Por lo tanto, el uso de muestras de saliva podría reducir el riesgo de transmisión nosocomial de 2019nCoV (25), eliminar el tiempo de espera para la recolección de muestras y, por lo tanto, los resultados estarían disponibles con mayor rapidez. Esto es especialmente importante en entornos clínicos ocupados donde el número de personal disponible es limitado. ${ }^{17}$

Las muestras de saliva se pueden proporcionar fácilmente solicitando a los pacientes que escupan en un frasco estéril, o en dispositivos de recolección de saliva disponibles en el mercado para una recolección segura y estéril sin comprometer la calidad y la cantidad, ${ }^{16,34}$ inclusive la posibilidad de una autocolección de saliva puede reducir considerablemente el riesgo de transmisión de COVID-19. ${ }^{10}$

Las pruebas de saliva podrían llegar a utilizarse en el campo, en los departamentos de emergencia, en 
clínicas médicas y dentales y, eventualmente, en el hogar. ${ }^{60}$

Este método de muestreo tiene la precisión y confiabilidad adecuadas con respecto al monitoreo de la carga viral del SARS-CoV-2 basado en la técnica de RTPCR. ${ }^{35}$

Hace algunas semanas un equipo de científicos de la UNAM diseñó un método para detectar al SARSCoV-2 mediante saliva: Dos mililitros de saliva son suficientes y eficaces, afirmó Susana López Charretón, del Departamento de Genética del Desarrollo y Fisiología Molecular del Instituto de Biotecnología (IBt), quien junto con su equipo han aplicado este proceso en pacientes de Morelos. Este procedimiento, además de disminuir el riesgo para el personal de salud y acelerar el diagnóstico, es 50 por ciento más económico que la recolección de muestras con hisopos en nariz y garganta. ${ }^{41}$

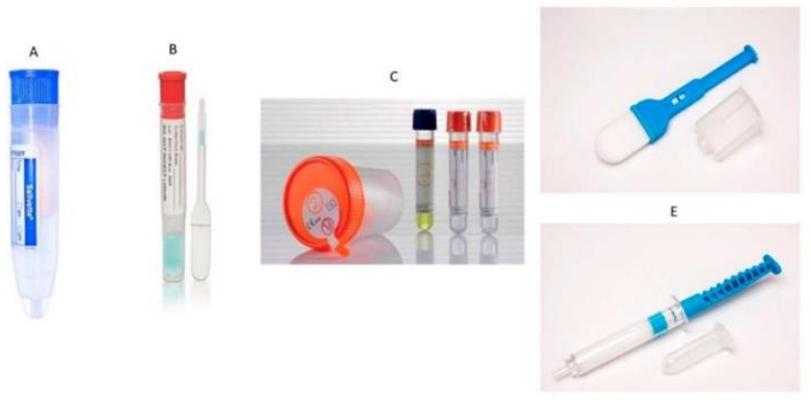

Figura 2: Representación de diferentes dispositivos de recolección de saliva utilizados en el muestreo de enfermedades infecto-contagiosas (34).

\section{Anticuerpos específicos contra virus en saliva}

Otro campo en el que la saliva puede tener un gran potencial para Covid-19 es la detección de inmunidad. Existe evidencia científica de que se pueden detectar anticuerpos específicos contra enfermedades infecciosas en la saliva (61). Las concentraciones salivales de IgG e IgM son mucho más bajas que en suero, aunque se ha sugerido que están en una proporción similar en relación con la proteína total en saliva que en suero. ${ }^{62}$ En el caso particular de la lgG, los perfiles de lgG en plasma y saliva son muy similares para un gran número de antígenos (63). Se ha planteado la hipótesis de que tanto la IgG como la lgM salivales se derivan de la sangre, mientras que la $\lg \mathrm{A}$ es producida principalmente por las glándulas salivales. $^{61}$
En especies animales, existe evidencia de que la saliva se puede utilizar para evaluar las respuestas de anticuerpos a las infecciones por coronavirus. ${ }^{43}$

Además, la saliva se ha utilizado en varias enfermedades virales de los seres humanos para la detección de inmunoglobulinas específicas frente a patógenos como la hepatitis $A, B$ y $C$, el virus de la inmunodeficiencia humana y el virus de la rubéola $(61,64,65) .{ }^{61,64,65}$

La detección de anticuerpos en la saliva podría usarse potencialmente para el control de Covid-19 como ha ocurrido con otras enfermedades infecciosas. Por ejemplo, para la infección por rubéola, el Servicio de Laboratorio de Salud Pública del Reino Unido ha ofrecido pruebas de fluidos orales para los casos notificados a la Oficina de Estadísticas Nacionales (ONS). En este programa, las muestras de saliva se recolectan entre dos y seis semanas después del inicio de los síntomas y se analizan para detectar IgM específicas del virus. Se ha demostrado que los análisis utilizados son más del $90 \%$ sensibles y específicos. ${ }^{62}$ Se podría esperar que la saliva tenga un valor similar en el diagnóstico y monitoreo de Covid-19.

Esto es particularmente interesante porque mientras no exista un tratamiento o vacuna eficaz y la transmisión de persona a persona deba minimizarse es de vital importancia tener la posibilidad de identificar individuos inmunes. ${ }^{66}$

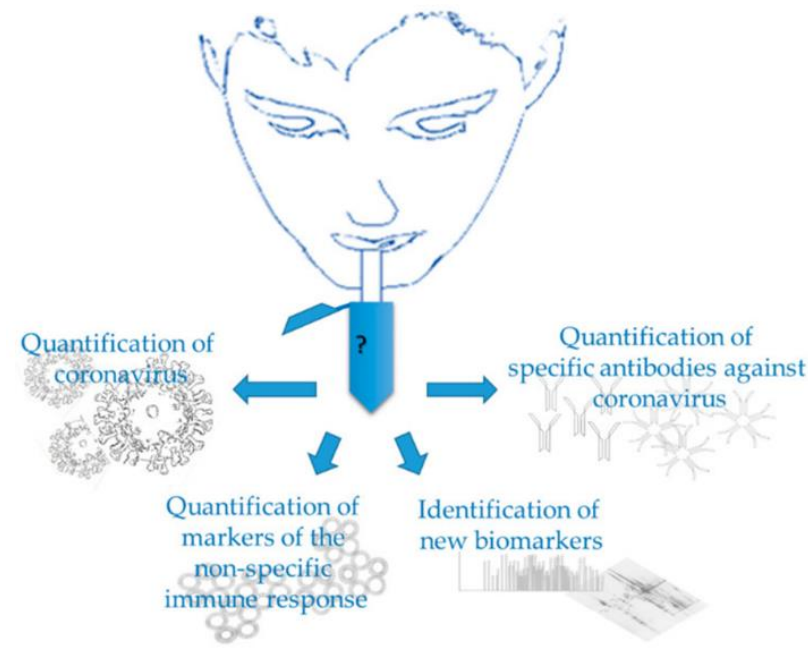

Figura 3: Uso de saliva para el diagnóstico de Covid19.67

\section{Conclusión}

La saliva tiene un papel fundamental en la transmisión de COVID-19 en la población, de hecho, estudios actuales han demostrado que la saliva podrá ser una alternativa validada no invasiva para el diagnóstico y el monitoreo de 
la carga viral de SARS-CoV-2, proporcionando una plataforma rentable y conveniente.

Debido al muestreo no invasivo, la facilidad de uso, la rentabilidad y el riesgo relativamente mínimo de infección cruzada, la saliva es un biofluido diagnóstico ideal; augurando un futuro auspicioso para el diagnóstico y seguimiento de la enfermedad, brindando una nueva alternativa diagnóstica que se suma a la batería de pruebas que se pueden realizar a un paciente sospechoso, de modo de detectar la enfermedad de forma rápida y segura, para comenzar con su respectivo período de aislamiento.

En este momento, en esta situación de pandemia controlable, todos los centros de investigación, agencias de salud y proveedores de atención médica deben explorar la oportunidad de diagnóstico y desarrollar rápidamente ensayos, que garanticen una detección temprana y segura, que ayude a prevenir y contener cuando los primeros brotes sean detectados.

\section{Referencias}

[1] Meng L, Hua F, Bian Z. Coronavirus Disease 2019 (COVID-19): Emerging and Future Challenges for Dental and Oral Medicine. J Dent Res. 2020 May;99(5):481-7.

[2] Melián-Rivas A, Calcumil-Herrera P, Boin-Bakit C, CarrascoSoto R. Detección de COVID -19 (SARS-CoV-2) Mediante la Saliva: Una Alternativa Diagnóstica poco Invasiva. Int $\mathrm{J}$ Odontostomatol..2020;14(3):316-20.

[3] Singhal T. A Review of Coronavirus Disease-2019 (COVID-19). Indian J Pediatr 2020;87:281-6.

[4] Rodriguez-Morales AJ, Cardona-Ospina JA, Gutiérrez-Ocampo E, Villamizar-Peña R, Holguin-Rivera Y, Escalera-Antezana JP, et al. Clinical, laboratory and imaging features of COVID-19: A systematic review and meta-analysis. Travel Med Infect Dis. 2020;34:101623.

[5] Van Doremalen N, Bushmaker T, Morris DH, Holbrook MG, Gamble A, Williamson BN, et al. Aerosol and surface stability of SARS-CoV-2 as compared with SARS-CoV-1. N Engl J Med. 2020;382:1564-7.

[6] Chin AWH, Chu JTS, Perera MRA, Hui KPY, Yen H-L, Chan $\mathrm{MCW}$, et al. Stability of SARS-CoV-2 in different environmental conditions. The Lancet Microbe. 2020;1(1):e10.

[7] Peng X, Xu X, Li Y, Cheng L, Zhou X, Ren B. Transmission routes of 2019-nCoV and controls in dental practice. Vol. 12, Int J Oral Sci. 2020; 12:9.

[8] Guan W, Ni Z, Hu Y, Liang W, Ou C, He J, et al. Clinical Characteristics of Coronavirus Disease 2019 in China. N Engl J Med. 2020;382(18):1708-20.

[9] COVID-19. Available from: https://www.entuk.org/covid-19

[10] Sabino-Silva R, Jardim ACG, Siqueira WL. Coronavirus COVID-19 impacts to dentistry and potential salivary diagnosis. Vol. 24, Clin Oral Investig. 2020; 24:1619-21.
Chen N, Zhou M, Dong X, Qu J, Gong F, Han Y, et al. Epidemiological and clinical characteristics of 99 cases of 2019 novel coronavirus pneumonia in Wuhan, China: a descriptive study. Lancet.2020;395(10223):507-13.

[12] Huang C, Wang Y, Li X, Ren L, Zhao J, Hu Y, et al. Clinical features of patients infected with 2019 novel coronavirus in Wuhan, China. Lancet. 2020;395(10223):497-506.

[13] Wang D, Hu B, Hu C, Zhu F, Liu X, Zhang J, et al. Clinical Characteristics of 138 Hospitalized Patients with 2019 Novel Coronavirus-Infected Pneumonia in Wuhan, China. JAMA - J Am Med Assoc.2020;323(11):1061-9.

[14] Yang Y, Lu Q, Liu M, Wang Y, Zhang A, Jalali N, et al. Epidemiological and clinical features of the 2019 novel coronavirus outbreak in China. medRxiv. 2020;2020.02.10.20021675.

[15] Nishiura H, Kobayashi T, Miyama T, Suzuki A, Jung S mok, Hayashi K, et al. Estimation of the asymptomatic ratio of novel coronavirus infections (COVID-19).J. Infect. Dis.2020;94(2):154-5.

[16] Azzi L, Carcano G, Gianfagna F, Grossi P, Gasperina DD, Genoni A, et al. Saliva is a reliable tool to detect SARS-CoV-2. J Infect. 2020;81(1):e45-50.

[17] Chan JFW, Yuan S, Kok KH, To KKW, Chu H, Yang J, et al. A familial cluster of pneumonia associated with the 2019 novel coronavirus indicating person-to-person transmission: a study of a family cluster. Lancet. 2020;395(10223):514-23.

[18] Del Rio C, Malani PN. 2019 Novel Coronavirus - Important Information for Clinicians.JAMA.2020;323: 1039-40.

[19] Zerón A. El coronavirus se ha hecho viral. Rev ADM. 2020;77(2):58-61.

[20] Xiao F, Tang M, Zheng X, Liu Y, Li X, Shan H. Evidence for Gastrointestinal Infection of SARS-CoV-2. Gastroenterology. 2020;158(6):1831-1833.e3.

[21] Xia J, Tong J, Liu M, Shen Y, Guo D. Evaluation of coronavirus in tears and conjunctival secretions of patients with SARS-CoV2 infection. J Med Virol. 2020;92(6):589-94.

[22] Holshue ML, DeBolt C, Lindquist S, Lofy KH, Wiesman J, Bruce $\mathrm{H}$, et al. First case of 2019 novel coronavirus in the United States. N Engl J Med. 2020;382(10):929-36.

[23] Young BE, Ong SWX, Kalimuddin S, Low JG, Tan SY, Loh J, et al. Epidemiologic Features and Clinical Course of Patients Infected with SARS-CoV-2 in Singapore. JAMA. 2020;323(15):1488-94.

[24] To KKW, Tsang OTY, Leung WS, Tam AR, Wu TC, Lung DC, et al. Temporal profiles of viral load in posterior oropharyngeal saliva samples and serum antibody responses during infection by SARS-CoV-2: an observational cohort study. Lancet Infect Dis. 2020;20(5):565-74.

[25] To KKW, Tsang OTY, Yip CCY, Chan KH, Wu TC, Chan JMC, et al. Consistent Detection of 2019 Novel Coronavirus in Saliva. Clin Infect Dis. 2020;71(15):841-3.

[26] Kim Y Il, Kim SG, Kim SM, Kim EH, Park SJ, Yu KM, et al. Infection and Rapid Transmission of SARS-CoV-2 in Ferrets. Cell Host Microbe . 2020;27(5):704-709.e2. 
Morales Espinosa R, La ER. La Saliva como Biomuestra para Diagnóstico de Infección por SARS-CoV-2: Una Revisión Saliva as a Bio-sample for Diagnosis SARS-CoV-2 Infection: A review. Vol. 14, Int. J. Odontostomat. 2020; 14(3):327-330.

[28] Tyrrell DA, Bynoe ML. Cultivation of viruses from a high proportion of patients with colds. Lancet. 1966;1(7428):76-7.

[29] Zhou P, Yang X Lou, Wang XG, Hu B, Zhang L, Zhang W, et al A pneumonia outbreak associated with a new coronavirus of probable bat origin. Nature. 2020;579(7798):270-3.

[30] Zhang T, Wu Q, Zhang Z. Probable Pangolin Origin of SARSCoV-2 Associated with the COVID-19 Outbreak. Curr Biol. 2020;30(7):1346-1351.e2.

[31] Lu R, Zhao X, Li J, Niu P, Yang B, Wu H, et al. Genomic characterisation and epidemiology of 2019 novel coronavirus: implications for virus origins and receptor binding. Lancet 2020;395(10224):565-74.

[32] Ksiazek TG, Erdman D, Goldsmith CS, Zaki SR, Peret T, Emery $\mathrm{S}$, et al. A Novel Coronavirus Associated with Severe Acute Respiratory Syndrome. N Engl J Med. 2003;348(20):1953-66.

[33] Zaki AM, Van Boheemen S, Bestebroer TM, Osterhaus ADME, Fouchier RAM. Isolation of a novel coronavirus from a man with pneumonia in Saudi Arabia. N Engl J Med. 2012;367(19):181420.

[34] Khurshid Z, Yahya F, Asiri I, Al Wadaani H. Human Saliva: NonInvasive Fluid for Detecting Novel Coronavirus (2019-nCoV). Int. J. Environ. Res. Public Health.2020 ;17(7): 2225.

[35] Fakheran O, Dehghannejad M, Khademi A. Saliva as a diagnostic specimen for detection of SARS-CoV-2 in suspected patients: A scoping review Infect. Dis. Poverty.2020; 9:7.

[36] Kwon KT, Ko JH, Shin H, Sung M, Kim JY. Drive-through screening center for covid-19: A safe and efficient screening system against massive community outbreak. J Korean Med Sci. 2020;35(11):10.

[37] Xu R, Cui B, Duan X, Zhang P, Zhou X, Yuan Q. Saliva: potential diagnostic value and transmission of 2019-nCoV. Int J Oral Sci. 2020;12(1):1-6.

[38] Fang Z, Zhang Y, Hang C, Ai J, Li S, Zhang W. Comparisons of viral shedding time of SARS-CoV-2 of different samples in ICU and non-ICU patients. J. Infect.2020;81:147-78.

[39] Humphrey SP, Williamson RT. A review of saliva: Normal composition, flow, and function. $\mathrm{J}$ Prosthet Dent 2001;85(2):162-9.

[40] Baghizadeh Fini M. Oral saliva and COVID-19 .Oral Oncol. 2020;108: 104821 .

[41] Moreno-Contreras J, Espinoza MA, Sandoval-Jaime C, CantúCuevas MA, Barón-Olivares H, Ortiz-Orozco OD, et al. Saliva Sampling and Its Direct Lysis, an Excellent Option To Increase the Number of SARS-CoV-2 Diagnostic Tests in Settings with Supply Shortages. J. Clin. Microbiol. 2020;58(10)

[42] Procop GW, Shrestha NK, Vogel S, Van Sickle K, Harrington S, Rhoads DD, et al. A Direct Comparison of Enhanced Saliva to Nasopharyngeal Swab for the Detection of SARS-CoV-2 in Symptomatic Patients. J Clin Microbiol. 2020;16.
[43] Bjustrom-Kraft J, Woodard K, Giménez-Lirola L, Rotolo M, Wang C, Sun Y, et al. Porcine epidemic diarrhea virus (PEDV) detection and antibody response in commercial growing pigs. BMC Vet Res. 2016;12(1):1-8.

[44] Niederwerder MC, Nietfeld JC, Bai J, Peddireddi L, Breazeale B, Anderson J, et al. Tissue localization, shedding, virus carriage, antibody response, and aerosol transmission of Porcine epidemic diarrhea virus following inoculation of 4-week-old feeder pigs. J Vet Diagnostic Investig. 2016 ;28(6):671-8.

[45] Khurshid Z, Zafar M, Khan E, Mali M, Latif M. Human saliva can be a diagnostic tool for Zika virus detection. J. Infect. Public Health. 2019;12:. 601-4.

[46] Boppana SB, Ross SA, Shimamura M, Palmer AL, Ahmed A, Michaels MG, et al. Saliva polymerase-chain-reaction assay for cytomegalovirus screening in newborns. $\mathrm{N}$ Engl $\mathrm{J}$ Med.2011;364(22):2111-8.

[47] Khan R, Khurshid Z, Yahya Ibrahim Asiri F. Advancing Pointof-Care (PoC) Testing Using Human Saliva as Liquid Biopsy. Diagnostics. 2017;7(3):39.

[48] Carbone Z, Haydee CN, González ; Mercedes M, Martínez ;, Elena S. La saliva: una mirada hacia el diagnóstico. RAAO.2016;15(2): 39-43.

[49] Tvarijonaviciute A, Martinez-Lozano N, Rios R, Marcilla de Teruel MC, Garaulet M, Cerón JJ. Saliva as a non-invasive tool for assessment of metabolic and inflammatory biomarkers in children. Clin Nutr.2020;39(8):2471-8.

[50] Centro de Protección de la Salud - Enfermedad por coronavirus (COVID-19) - Cartas a los médicos. Available from: https://www.chp.gov.hk/en/miniweb/letters/100063.html

[51] Wang WK, Chen SY, Liu IJ, Chen YC, Chen HL, Yang CF, et al. Detection of SARS-associated coronavirus in throat wash and saliva in early diagnosis. Emerg Infect Dis. 2004;10(7):1213-9.

[52] To KK, Lu L, Yip CC, Poon RW, Fung AM, Cheng A, et al. Additional molecular testing of saliva specimens improves the detection of respiratory viruses. Emerg Microbes Infect. 2017 Jun $7 ; 6(6): e 49$

[53] Fang Y, Zhang H, Xie J, Lin M, Ying L, Pang P, et al. Sensitivity of chest CT for COVID-19: Comparison to RT-PCR.Radiology. 2020;296: E115-7.

[54] Zheng S, Yu F, Fan J, Zou Q, Xie G, Yang X, et al. Saliva as a Diagnostic Specimen for SARS-CoV-2 by a PCR-Based Assay: A Diagnostic Validity Study. SSRN Electron J. 2020 ;18.

[55] Chen L, Zhao J, Peng J, Li X, Deng X, Geng Z, et al. Detection of 2019-nCoV in Saliva and Characterization of Oral Symptoms in COVID-19 Patients. SSRN Electron J. 2020;30.

[56] Zheng S, Fan J, Yu F, Feng B, Lou B, Zou Q, et al. Viral load dynamics and disease severity in patients infected with SARSCoV-2 in Zhejiang province, China, January-March 2020: Retrospective cohort study. BMJ . 2020;369:8.

[57] Williams E, Bond K, Zhang B, Putland M, Williamson DA. Saliva as a noninvasive specimen for detection of sars-cov-2. J. Clin. Microbiol. 2020;58: 5.

[58] Yoon JG, Yoon J, Song JY, Yoon SY, Lim CS, Seong H, et al. Clinical significance of a high SARS-CoV-2 viral load in the 
Saliva. J Korean Med Sci.2020;35(20).

[59] Han MS, Seong MW, Heo EY, Park JH, Kim N, Shin S, et al. Sequential analysis of viral load in a neonate and her mother infected with SARS-CoV-2. Clin Infect Dis. 2020;4.

[60] Corstjens PLAM, Abrams WR, Malamud D. Detecting viruses by using salivary diagnostics. J Am Dent Assoc. 2012;143:12S-18S.

[61] Parry J V., Perry KR, Mortimer PP. Sensitive assays for viral antibodies in saliva: an alternative to tests on serum. Lancet $.1987 ; 330(8550): 72-75$.

[62] McKie A, Vyse A, Maple C. Novel methods for the detection of microbial antibodies in oral fluid. Lancet Infect. 2002;2(1):18-24.

[63] Hettegger P, Huber J, Paßecker K, Soldo R, Kegler U, Nöhammer $\mathrm{C}$, et al. High similarity of $\operatorname{IgG}$ antibody profiles in blood and saliva opens opportunities for saliva based serology. PLoS One;14(6):1-17.

[64] Mortimer PP, Parry J V. Detection of antibody to HIV in saliva: a brief review. Clin Diagn Virol.1994;2(4-5):231-43.

[65] González V, Martró E, Folch C, Esteve A, Matas L, Montoliu A, et al. Detection of hepatitis C virus antibodies in oral fluid specimens for prevalence studies. Eur J Clin Microbiol Infect Dis. 2008;27(2):121-6.

[66] Flodgren GM. Immunity after SARS-CoV-2 infection, 1st update - a rapid review. Lancet Infect. Dis.2020;23:23.

[67] Ceron J, Lamy E, Martinez-Subiela S, Lopez-Jornet P, CapelaSilva F, Eckersall P, et al. Use of Saliva for Diagnosis and Monitoring the SARS-CoV-2: A General Perspective. J Clin Med. 2020;9(5):1491. 